\title{
MODE DE TRANSMISSION DE LA MALADIE DE C. CHAGAS
}

\author{
Par E. BRUMPT
}

Depuis quelques années, des faits expérimentaux ou accidentels et de nombreuses observations cliniques ont établi que la maladie de C. Chagas peut être due à la contamination de la muqueuse oculaire par les déjections ou le produit de broyat de triatomes hébergeant Trypanosoma cruzi.

Depuis notre première publication (1912), où nous avions montré qu'un Cercopithecus ruber (395/II), ayant résisté à de nombreuses piqûres de Triatoma megista, avait pris une infection mortelle après dépôt sur la muqueuse oculaire de déjections de triatomes riches en Trypanosoma cruzi, nous avons souvent enregistré des faits identiques. En particulier, en utilisant des Lemur mongoz (Exp. 413 et $434 / \mathrm{II}$, novembre 1912), ainsi qu'un certain nombre de jeunes animaux, principalement des chats et des chiens, nous avons pu renouveler cette observation.

Jusqu'à ce jour, nous n'avons observé, ni dacryocystite, ni dacryo-adénite chez les animaux signalés ci-dessus, infectés par la voie oculaire, ainsi que chez un jeune porcelet dont l'infection a été établie par le xénodiagnostic.

Si la contamination de la muqueuse oculaire est facile à observer, il n'en est pas moins vrai que l'infection peut se produire par d'autres portes d'entrée, telles que la muqueuse nasale ou buccale, ou en un point quelconque du corps, à la suite de lésions de grattage, déterminées par des ongles souillés de déjections de triatomes ou d'un broyat accidentel d'un de ces insectes. E. Dias (1934) a, en effet, constaté qu'au Brésil, la literie des habitants des zones à maladie de Chagas était souvent souillée de déjections de triatomes et de taches de sang provenant de leur écrasement volontaire ou non pendant la nuit.

Comme nous ne savons pas quels symptômes présentent les sujets infectés par un autre point que la muqueuse oculaire, il est difficile d'établir dans quelle proportion l'infection se produit par cette dernière voie.

En dehors du rôle habituel des réduvidés hématophages répartis

Annales de Parasitologie, T. XVII, N $4 .-1^{\text {er }}$ juillet 1939 , p. $320-331$. 
dans les genres Triatoma, Rhodnius et Eratyrus (1), il est probable que la maladie de Chagas peut également se contracter par la dissection, dans un but culinaire ou taxidermique, des tatous, sarigues ou animaux divers constituant des réservoirs naturels de Trypanosoma cruzi.

Deux faits permettent d'affirmer que les nombreux cas aigus avec

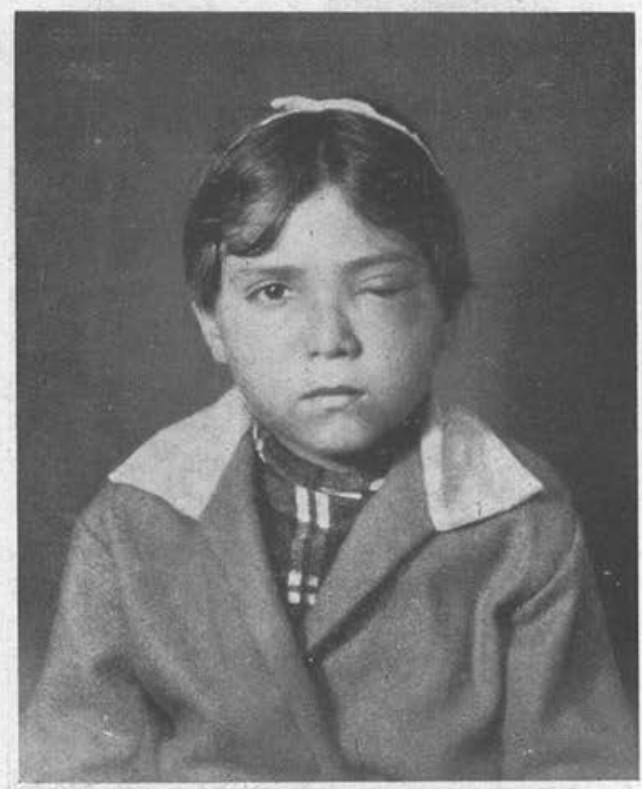

Fıg. 1. - Forme aiguë de la maladie de Chagas, œdème débutant par l'œil gauche qui a servi de porte d'entrée au Trypanosoma cruzi des déjections de triatome. Cliché du Prof. R. Talice.

œedème unilatéral de la face (fig. 1 et 2), observés, après Chagas, depuis 1934, par Mazza, Romaña et Talice, ont eu comme porte d'entrée la voie oculaire. Le premier fait est une expérience d'Evandro Chagas (1935), qui a déterminé une infection chez un homme en déposant sur la conjonctiye des déjections de triatomes riches en formes métacycliques du $T$. cruzi. Le second fait, qui confirme le précédent, est celui qui a été publié par A. Herr et L.-C. Brumpt

(1) A ces trois genres, il convient d'ajouter un hémiptère hématophage de petite taille, Clerada apicicornis, trouvé dans les chaumières de l'Ámazonie (Brésil) par L. de Castro Ferreira et L. Deane (1938) et que ces auteurs ont pu infecter expérimentalement par Trypanosoma cruzi.

Annales de Parasitologie, T. XVII, N ${ }^{\circ} 4 .-1^{\text {er }}$ juillet 1939. 
(1939), concernant une infection accidentelle de laboratoire dont l'œil droit avait été la porte d'entrée. Les symptômes observés dans ces deux cas étaient identiques à ceux des formes aiguës spontanées étudiées par les auteurs sud-américains. Il est done permis d'affirmer que l'infection naturelle se produit souvent par la contamination de la muqueuse oculaire, et cette constatation

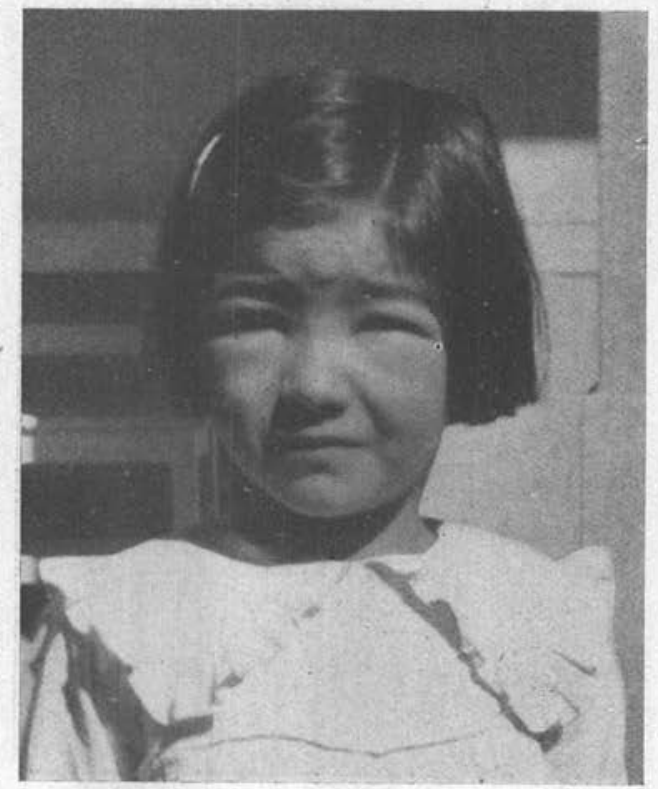

Fı́. 2. - Forme aiguë de la maladie de Chagas, cedème bilatéral avec prédominance à gauche. Cliché du $\mathrm{D}^{r} \mathrm{~A}$. Ruchelli.

est d'un intérêt épidémiologique tout particulier, en ce qui concerne le mode de transmission de la maladie de Chagas.

En effet, des cas de cette trypanosomose surviennent parfois chez des sujets plus ou moins âgés qui vivent depuis leur naissance dans des chaumières où abondent des triatomes, infectés fréquemment dans plus de 50 p. 100 des cas. Nous pouvons done admettre que certains sujets piqués chaque jour pendant, leur sommeil par trois, quatre, dix triatomes infectés et ayant subi ainsi, sans dommage, des milliers de piqûres au cours de leur existence, sont capables de prendre la maladie le jour où, accidentellement, en se frottant les yeux, ils y introduisent les déjections virulentes déposées sur leurs joues ou leurs paupières (fig. 3). Ces 
faits sont du plus haut intérêt aits montrent que Pinfection contaminative est de beaucoup la plus importante dans la nature et ils confirment toutes les expériences que nous avions publiées de 1912 à 1914 et que nous avons résumées dans les éditions successives de notre Précis de Parasitologie.

Il est bien certain que, si les germes de la maladie étaient inoculés avec le liquide salivaire des insectes, comme dans le cas du paludisme ou de la maladie du sommeil, par exemple, on aurait quelque peine à expliquer ces faits épidémiologiques.

A la lumière de ces observations récentes, expérimentales, acci-

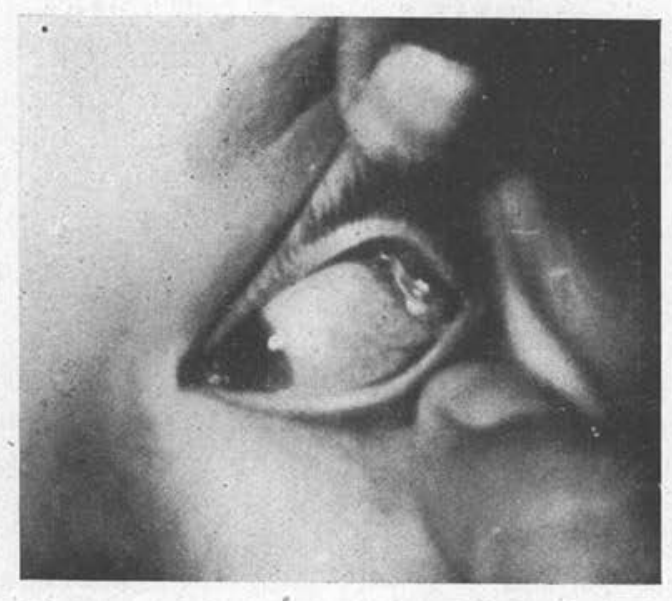

Fì 3. 3. - Forme aiguë de la maladie de Chagas. Dacryo-adénite de l'œil gauche. Cliché du Prof. A. Talice.

dentelles et cliniques, il est intéressant de passer en revue les expériences qui ont été effectuées dans différents pays en vue d'établir par quel mécanisme les vertébrés peuvent contracter la maladie de Chagas.

Deux hypothèses ont été émises relativement à ce mode de transmission : celle de C. Chagas, suivant laquelle l'infection humaine serait due à l'inoculation passive de trypanosomes par la piqûre de l'insecte vecteur, et la nôtre, qui prévoit la pénétration active des trypanosomes métacycliques infectieux chez l'homme par les muqueuse soit à la faveur d'une égratignure, soit même par la peau intacte humide.

C. Chagas, influencé par les travaux de Schaudinn sur le cycle évolutif des trypanosomes de la chevêche, avait décrit en 1909 
et 1911, un cycle évolutif complexe du $T$. cruzi. Ce flagellé se développerait suivant deux types chez les triatomes. Le premier type, asexué, prendrait naissance dans l'intestin moyen et donnerait des formes Crithidia. Le second type, sexué, aboutirait à la formation de trypanosomes se localisant dans les glandes salivaires. Cellesci étaient envahies par des trypanosomes passant dans la cavité générale de l'insecte où Chagas les avait aperçues deux fois. Ce même auteur avait observé une infection des glandes salivaires étudiée sur frottis, dans trois cas.

En 1912, en collaboration avec P. da Silva, nous avons établi, pour la première fois, que les déjections des triatomes infectés renfermaient de nombreux flagellés qui, inoculés dans le péritoine de souris, produisaient une infection typique. Quelques semaines plus tard, nous décrivions le cycle évolutif complet du parasite chez l'insecte vecteur. Ce cycle était totalement différent de celui des trypanosomes de la maladie du sommeil chez les tsétsés, et était superposable à celui des trypanosomes non pathogènes des rongeurs chez les puces, dont le cycle avait été établi par Minchin et Thomson en 1910. Le cycle du $T$. cruzi s'effectuait uniquement dans l'intestin et les formes infectieuses se formaient exclusivement dans la partie postérieure de cet organe. Les formes observées ne présentaient aucune différenciation sexuelle, mais montraient tous les types morphologiques que nous avions décrits en 1904, époque à laquelle, en étudiant l'évolution des trypanosomes de poissons et de batraciens chez les sangsues, nous avions établi pour la première fois le cycle évolutif d'un trypanosome et la formation des formes métacycliques infectieuses.

Nos recherches, concernant le cycle évolutif du $T$. cruzi et le mode d'infection des animaux de laboratoire avec ce parasite, ont été confirmées par de nombreux auteurs. Par contre, jusqu'à ce jour, aucun d'eux n'a rencontré, dans les glandes salivaires, les formes que Chagas avait signalées sur des frottis de ces organes, peut-être souillés par des flagellés de la cavité générale provenant eux-mêmes de quelque lésion accidentelle du tube digestif déterminée par la trompe d'un triatome cannibale ou par un traumatisme quelconque.

E. Dias a essayé d'infecter les glandes salivaires des triatomes en inoculant, dans la cavité générale de ces derniers, des $T$. cruzi qui, ainsi qu'il l'a démontré, s'y développent aussi normalement que dans le tube digestif. En aucun cas, cet auteur n'a constaté d'infection des glandes salivaires, ce qui prouve bien que ces organes n'exercent aucune attraction pour les trypanosomes à quelque degré d'évolution qu'ils soient. 
Nous croyons utile de donner ci-dessous, en suivant l'ordre chronologique, quelques indications sur les recherches expérimentales concernant le mode d'infection de l'homme et des animaux de laboratoire.

De 1909 à 1927, C. Chagas a obtenu des infections sans éviter la contamination par les déjections, en faisant piquer un oustiti (Callithrix penicillata) et quelques cobayes par des Triatoma megista, mais il a également enregistré, dans les mêmes conditions, de nombreux résultats négatifs qu'il attribue à l'absence de formes sexuées infectieuses dans les glandes salivaires des insectes vecteurs.

En 1912, en collaboration avec Piraja da Silva, nous avons obtenu des résultats négatifs en utilisant le chien et le cobaye et des Triatoma megista. Quelques mois plus tard (1912), nous avons déterminé une infection mortelle chez un singe (Cercopithecus ruber), qui avait résisté aux piqûres d'exemplaires de $T$. megista, en déposant des déjections de ces derniers insectes sur la muqueuse oculaire, qui n'a d'ailleurs présenté aucune lésion locale.

Magarine Torres (1913-1915), sur 18 expériences permettant d'éviter la contamination par les déjections des triatomes, a réussi à infecter, par piqûre de Triatoma megista, deux chats et un cobaye ; les 15 autres animaux : cobayes, chats, oustitis, sont restés indemnes.

Maggio et Rosenbusch (1915) n'ont rien obtenu chez de jeunes chiens et de jeunes cobayes piqués par des triatomes ( $T$. infestans) dont les déjections permettaient d'infecter sans difficulté ces mêmes animaux, ainsi que des souris et des rats.

Neiva et Penna (1916) ont essayé sans succès d'infecter de nombreux chiens, chats et cobayes en les faisant piquer par des $T$. megista dont les déjections étaient riches en trypanosomes. Cependant, en utilisant des triatomes de la même espèce provenant d'une localité de l'Etat de Minas, ils pensent avoir obtenu quelques résultats positifs chez le cobaye.

Ces mêmes auteurs ont réussi l'infection d'un cobaye en déposant des déjections de Triatoma sordida sur la conjonctive; huit jours plus tard, les trypanosomes étaient visibles dans le sang de l'animal.

Gaminara (1923) a obtenu l'infection de divers animaux, cobayes, chiens, chats et souris en leur injectant sous la peau des déjections de $T$. infestans et de $T$. rubrovaria. Les infections obtenues étaient en général faibles. Cet auteur a enregistré des échecs en faisant piquer un cobaye par trois exemplaires de $T$. rubrovaria 
dont les déjections ont infecté deux cobayes témoins, puis deux autres cobayes piqués par d'autres exemplaires de triatomes de la même espèce, dont les déjections renfermaient de nombreux flagellés.

Mühlens, Dios, Petrocchi et Zuccarini (1925) ont observé des Trypanosoma eruzi chez un cobaye et deux souris piqués par des Triatoma infestans. Ces divers animaux avaient été mis à l'abri d'une contamination possible par les déjections des insectes. Cependant, en utilisant les mêmes triatomes qui avaient infecté les trois animaux signalés ci-dessus, ces auteurs n'ont obtenu, dans la suite, que des résultats négatifs en utilisant des cobayes et des souris du même âge, ce qu'ils expliquaient en admettant l'état réfractaire de certains mammifères, hypothèse à laquelle nous ne pouvons souscrire, car tous les animaux de laboratoire sont réceptifs aux inoculations par passage de sang ou aux inoculations de déjections de triatomes.

Parreiras Horta (cité par Mühlens et coll., 1925), n'a jamais pu obtenir d'infection chez divers animaux de laboratoire en les faisant piquer par des triatomes présentant des trypanosomes dans leurs déjections.

En 1929, Niño, en faisant piquer un cobaye par des Triatoma infestans infectés, a enregistré un résultat négatif.

Clark et Dunn (1932), à Panama, infectent sept cobayes sur lesquels avaient été nourris des Triatoma geniculata, mais comme aucune précaution n'avait été prise pour éviter la contamination par les déjections, ces auteurs estiment ne pouvoir tirer aucune conclusion de leur expérience. La mêmé remarque peut être faite à l'occasion de l'infection d'un cobaye, piqué à Panama à plusieurs reprises, par un seul exemplaire d'Eratyrus cuspidatus (Dunn, 1934).

De 1932 à 1934, Dias, à qui nous devons le plus important travail sur ce sujet, n'a jamais obtenu d'infections en faisant piquer quatorze cobayes par cent-quatre exemplaires de Triatoma megista dont les déjections étaient infectieuses.

En utilisant des Triatoma protracta de Californie, Kofoid et Donat (1933) ont toujours enregistré des résultats négatifs par piqûre d'animaux réceptifs, alors que l'inoculation des déjections faisait régulièrement apparaitre des trypanosomes dans le sang.

Evandro Chagas (1935) a eu un résultat négatif en faisant piquer un homme volontaire par des Triatoma megista infectés.

En 1938, Cardoso, en utilisant, dans notre laboratoire, un élevage de Triatoma infestans et une souche de Trypanosoma cruzi d'ori- 
gine brésilienne, assez pathogène pour la souris, a obtenu une infection sur 10 souris piquées chacune par un seul exemplaire, dans des conditions écartant toute possibilité de contamination par les déjections des insectes. Cependant, une seconde expérience effectuée avec le triatome qui s'était montré infectieux une première fois, a été négative, ce qui permet d'admettre, dans le premier cas, une régurgitation des parasites de l'estomac suivant l'hypothèse de Dias et de Hoare.

Au cours d'un récent voyage au Mexique (1938), nous avons eu l'occasion d'isoler un certain nombre de souches de Trypanosoma cruzi provenant de diverses localités mexicaines, où leurs vecteurs habituels sont, ainsi que L. Mazzotti l'a établi en 1936, Triatoma phyllosoma et $T$. pallidipennis. L'une de ces souches, originaire de Tonala (Chiapas), était assez virulente pour la souris, et c'est cependant sans succès que nous avons fait piquer un jeune rat par plus de 50 nymphes de $T$. pallidipennis dont les déjections furent infectieuses pour un rat témoin du même âge.

D'autre part, les $\mathrm{D}^{r s}$ Denecke et von Haller (1939), expérimentant à notre laboratoire avec cinq souches que nous leur avions confiées, ont eu 50 résultats négatifs par piqùre d'un seul Triatoma pallidipennis ou d'un seul Rhodnius prolixus sur chaque animal, alors que tous les témoins ont toùs été infectés par les déjections de ces mêmes insectes.

Nous ne croyons pas utile de citer les expériences de nombreux auteurs qui ont confirmé nos premiers travaux, en montrant la facilité avec laquelle les trypanosomes métacycliques de triatomes passent à travers la muqueuse saine, la peau légèrememnt scarifiée et parfois même la peau saine de jeunes animaux. La plupart de ces faits sont résumés dans le travail de F.-A. Cardoso (1938).

Il résulte donc des expériences réalisées par Torres (1913-1915), Mühlens et ses collaborateurs, en 1925, puis par Cardoso (1938) dans des conditions qui semblent exclure toute contamination possible par les déjections de triatomes, que l'infection peut parfois être transmise par la piqûre de ces derniers, ce qui ne prouve d'ailleurs pas une infection des glandes salivaires. E. Dias (1934), puis Hoare (1934), admettent, en effet, une régurgitation éventuelle de trypanosomes conservés dans l'estomac des insecteurs vecteurs, où, d'après E. Dias, ils sont susceptibles de persister sans évoluer pendant au moins huit jours.

L'hypothèse de Dias et de Hoare est admissible, mais les recherches expérimentales entreprises sur mes conseils par Cardoso (1938) n'ont pu cependant la confirmer. Cet auteur a, en effet, 
disséqué 10 Triatoma infestans, infectés depuis plusieurs semaines, et en a inoculé le contenu stomacal à 10 souris qui sont restées indemnes, tandis que les 10 souris témoins inoculées avec les déjections de ces mêmes triatomes se sont toutes infectées.

Dans une analyse du travail de Cardoso, parue dans le Tropical Disease Bulletin (1939), Warrington Yorke admet la possibilité d'une contamination de la trompe des triatomes par leurs déjections renfermant des trypanosomes métacycliques infectieux. C'est une hypothèse intéressante que nous chercherons à vérifier expérimentalement, car, lorsque plusieurs triatomes sont dans un même tube, certains d'entre eux peuvent également souiller leur trompe par cannibalisme.

Il faudrait reprendre ces expériences avec différentes espèces de triatomes ayant donné la maladie par piqûre, infecter leurs descendants, toujours indemnes d'infection, avec ce même virus, sacrifier des exemplaires chaque jour et inoculer le contenu stomacal à des souris, afin d'établir la longévité des trypanosomes chez des animaux possédant peut-être accidentellement un milieu organique particulièrement favorable, transmis par voie héréditaire.

On pourrait encore admettre qu'en raison de tropismes anormaux, quelques trypanosomes métacycliques, au lieu de rester dans l'ampoule rectale, leur habitat normal, se dirigent vers les parties supérieures des voies digestives, d'où ils peuvent être inoculés par régurgitation.

Enfin, bien qu'aucun auteur n'ait revu, dans les glandes salivaires, les trypanosomes que C. Chagas y a signalés, ce que l'on sait des parasites erratiques ne permet pas de nier absolument une localisation salivaire accidentelle, déterminée, soit par un tropisme individuel de certains trypanosomes, soit par une attraction également accidentelle résultant des propriétés physico-chimiques des glandes salivaires de certains individus d'espèces diverses de triatomes. Cependant, un fait concluant, émanant des expériences de Mühlens, Dios, Petrocchi et Zuccarini (1925), puis de Cardoso (1938), c'est que des animaux qui ont donné une première fois une infection par piqûre ont été incapables de la transmettre une seconde fois, alors que l'intestin et les déjections de ces insectes renfermaient de nombreuses formes infectieuses. Ces faits démontrent donc le caractère fortuit des infections par piqûre dans les conditions expérimentales probablement assez différentes des conditions naturelles.

Les expériences les plus importantes à retenir sont celles de 
Denecke et von Haller (1939), qui ont fait piquer des souris par des insectes à déjections infectieuses, isolés depuis plusieurs semaines de leurs congénères, afin d'éviter l'infection de leur trompe par cannibalisme et ayant eu peu de chances d'infecter cet organe avec les trypanosomes métacyoliques de leurs déjections. Les expériences de ces auteurs seront poursuivies dans notre laboratoire et nous tenterons d'établir la longévité, en milieu humide, des trypanosomes métacycliques des déjections entre les pièces buccales de la trompe des triatomes, ainsi que la durée, dans ce même organe, des trypanosomes que ces insectes auraient pu puiser par cannibalisme chez un triatome infecté. Ces expériences permettront d'établir le bien-fondé de l'hypothèse de W. Yorke.

D'autre part, des études seront entreprises en vue d'établir la longévité des formes sanguicoles dans l'estomac des triatomes, ainsi que l'éventualité de la régurgitation chez des animaux isolés depuis plus ou moins longtemps, observations qui permettraient de vérifier l'hypothèse formulée par E. Dias et Hoare.

Quels que soient les résultats de ces recherches, qu'il sera, malgré tout, difficile de comparer avec ce qui se passe dans la nature, un fait demeure, dès maintenant, bien acquis, c'est que toute l'épidémiologie de la maladie de Chagas vient confirmer l'hypothèse de l'infection normale de l'homme par contamination des muqueuses ou par des lésions de grattage et semble infirmer celle de l'infection par piqûre d'insectes hébergeant le Trypanosoma cruzi dans leur tube digestif.

\section{Bibliographie}

Brumpt (E.). - Le Trypanosoma cruzi évolue chez Conorhinus megistus, Cimex lectularius, Cimex boueti et Ornithodorus moubata. Cycle évolutif de ce parasite. Bull. Soc. path. exot., V, 1912, p. 360.

Pénétration du Schizotrypanum cruzi à travers la muqueuse oculaire saine. Bull. Soc. path. exot., V, 1912, 724.

Etudes expérimentales de la trypanosomose américaine de C. Chagas. Bull. Acad. Méd., LXVII, 11 juin 1912, p. 431.

Evolution de Trypanosoma lewisi, nabiasi, blanchardi chez les puces et les punaises. Transmission par les déjections. Comparaison avec T. cruzi. Bull. Soc. Path. Exot., VI, 1913, 167.

Immunité partielle dans les infections à Trypanosoma cruzi, transmission de ce trypanosome par Cimex rotundatus. Rôle régulateur des hôtes intermédiaires. Passage à travers la peau. Bull. Soc. path. exot., VI, $1913 a, 173$.

Le xénodiagnostic. Applications au diagnostic de quelques affections parasitaires et en particulier à la trypanosomose de C. Chagas. Bull. Soc. path. exot., VII, 1914, 706.

Précis de Parasitologie, $1^{\text {ro }}$ édit. $1910 ; 2^{\circ}$ édit. $1913 ; 3^{\circ}$ édit. 1922 ; $4^{\circ}$ édit. 1927 ; 5 édit. 1936, Paris, Masson et Cie. 
Brumpt (E.). - Maladie de Chagas au Brésil. Mode de transmission, origine, conditions qui déterminent sa répartition actuelle. Bull. Acad. Méd., LXXXI, 1919, p. 251.

- Faits expérimentaux et cliniques concernant le mode de transmission de la maladie de Chagas ou trypanosomose américaine. C.R. Soc. Biol., CXXX, mars 1939 , p. 1197.

- La maladie de C. Chagas. Exemple d'infection très répandue, considérée jusqu'à ce jour comme rare faute d'enquêtes épidémiologiques méthodiques. Presse Médicale, $\mathrm{n}^{\circ}$ 50, 24 juin 1939, p. 1013.

- Quelques faits épidémiologiques concernant la maladie de C. Chagas. Presse Médicale, $\mathrm{n}^{\circ}$ 54, 8 juillet 1939.

Brumpt (E.), Mazzotri (L.) et Brusipt (L.-C.). - Enquêtes épidémiologiques sur la maladie de C. Chagas au Mexique. Réduvidés vecteurs. Animaux réservoirs de virus. Cas humains. Ann. Parasit., XVII, 1939, p. 299-312.

Brumpt (R.) et da Silva. - Existence du Schizotrypanum cruzi Chagas, 1909, à Bahia (Matta de São João). Biologie du Conorhinus megistus. Bull. Soc. Path. Exot., V, 1912, p. 22.

Cardoso (F. A.). - Sur le mécanisme de la transmission de la maladie de Chagas. Ann. Parasit., XVI, 1938, p. 341.

Castro Ferreira (L. de) et Deane (L.). - Infeção experimental do * Clerada apicicornis 》Signoret, 1863, pelo «Schizotrypanum cruzi 》 (Chagas, 1909). Nota previa. Brasil-Medico, LII, 1938, p. 1181.

Chragas (C.). - Neue Trypanosomen. (Vorläufige Mitteilung). Arch. f. Schiffs-u. Trop.-Hyg., XIII, 1909, p. 120.

- Quelques aspects évolutifs du Trypanosoma cruzi dans l'insecte transmetteur. C.R. Soc. Biol., XCVII, 1927, p. 829.

Chagas (E.-V.). - L'infection expérimentale chez l'homme par le Schizotrypanum cruzi. C.R. Soc. Biol., CXVIII, 1935, pp. 290-292.

- Infection expérimentale par le Schizotrypanum cruzi chez l'homme. C.R. Soc. Biol., CXVIII, 1935, p. 718.

Clark (H. C.) et Dunn (L. H.). - Experimental studies on Chagas Disease in Panama. Amer. Jl. of Trop. Med., XII, 1932, p. 49-77.

Denecke (K.) et von Haller (E.). - Recherches expérimentales sur le mode de transmission et le cours de l'infection par Trypanosoma cruzi chez les souris. Ann. Parasit., XVII, 1939, p. 313-319.

Dias (E.). - O Trypanosoma cruzi pode evoluir na cavidade general do Triatoma megista. Mem. Inst. Oswaldo Cruz, XXVI, 1932, p. 83.

Sur les déjections du Triatoma megista. Aspects du Trypanosoma cruzi

que l'on y rencontre. C.R. Soc. Biol., CXI, 1932a, p. 486 .
Expériences sur la transmission du Trypanosoma cruzi de l'insecte au vertébré. C.R. Soc. Biol., CXI, $1932 b$, p. 490.

Estudos sobre o Schizotrypanum cruzi. Mem. Inst. Oswaldo Cruz., XXVIII, 1934, p. 1.

Duns (L. H.). - Notes on the Reduvid Bug, Eratyrus cuspidatus Stäl, naturally infected with Trypanosoma cruzi Chagas found in Panama. Am. Journ. Trop. Med., XIV, 1934, p. 291.

Gaminara (A.). - Estudio experimental sobre Schizotrypanum cruzi y enfermedad de Chagas en el Uruguay. Anales Fac. de Med., Montevideo, mai 1923.

Herr (Annette) et Brumpt (L.-C.). - Un cas aigu de maladie de Chagas contracté accidentellement au contact de triatomes mexicains. Obser- 
vation et courbe fébrile. Bull. Soc. Path. Exot., XXXII, 10 mai 1939, p. 565.

HoAre (C. A.). - The transmission of Chagas disease. A critical review. Trop. Dis. Bull., XXXI, 1934, pp. 757-762.

KofoIn (C. A.) et Donat (F.). - South American trypanosomiasis of the human type. - Occurrence in mammals of the United-States. California * Western Med., XXXVIII, 1933, p. 1.

Maggio (C.) et Rosenbusch (F.). - Studien über die Chagaskrankheit in Argentinien und die Trypanosomen der Vinchucas (Wanzen, Triatoma infestans). Centrlbl. $f$. Bakt., Orig., LXXVII,-1916, p. 40.

Mazza (S.). - Sobre el valor del edemia palpebral de un solo lado para el diagnostico de forma aguda de la enfermedad de Chagas. Novena Reun. Soc. Argent. Pato. Reg., $\mathrm{n}^{\circ} 110,1935$.

Nota sopra el primer centenar de formas agudas de enfermedad de Chagas comprobadas en la Republica. Prensa Med. Argent., 19 août 1936.

Nuestra experienca sobre la enfermedad de Chagas en la Republica Argentina. Festchrift Nocht, 1937.

Nota a proposito de 240 cases agudos de enfermedad de Chagas comprobados en el pais par la M.E.P.R.A. Prensa Med. Argent., 21 juillet 1937.

Mazzotri (L.). - Investigacion sobre la existencia de la enfermedad de Chagas en el pais. Demostracion de tripanosomas en los zeduvideos trasmisores. Medicina, Mexico, XVI, 1936, p. 584-585.

Mühlens (P.), Dios (R.), Petrocchi (J.) et Zuccarini (J.). - Trypanosomiasis humana. Rev. Inst. Bact. Buenos Aires, IV, 1925, p. 290-323.

Neiva (A.) et Penna (B.). - Viajem cientifica pelo Norte da Bahia, sudoeste de Pernambuco, sul do Piauhi et de norte à sul de Goiaz. Mem. do Inst. Oswaldo Cruz, 1916, p. 117 et p. 120.

Nıño (F.). - Contribucion al estudio de la enfermedad de Chagas o trypanosomiasis americana en la Republica Argentina. Buenos Aires, Imprenta de la Universidad, 1929.

TAlice (R. V.). - Epidemiologia de la enfermedad de Chagas en el Uruguay. Arch. Urug. Med. Cir., XIII, 1938, p. 45.

Recherches personnelles sur une endémie ancienne et pourtant méconnue en Uruguay, la maladie de Chagas ou trypanosomiase américaine. Presse Médicale, 1939, p. 854.

Torres (M.). - Molestia de «Carlos Chagas ». Transmissão do T. cruzi pela picada da T. megista (Nota preliminar). Brasil-Medico, XXVII, 1913, 321.

Alguns fatos que interessam à epidemiologia da molestia de Chagas. Mem. Inst. Oswaldo Cruz, VII, 1915, 120.

YoRKe (W.). - Chagas disease. A critical review. Trop. Dis. Bull., XXIV, 1937, p. 275 .

Analyse du travail de Cardoso. Ibid., XXXVI, 1939, p. 229.

Institut de Parasitologie de la Faculté de médecine de Paris

(Directeur: Prof. E. Brumpt). 